\title{
NON-CONTRAST MAGNETIC RESONANCE UROGRAPHY- A PROSPECTIVE STUDY
}

\author{
J. S. Aswini Jyothi' ${ }^{1}$ M. Vijaya Kumari², N. Jayalatha ${ }^{3}$ \\ ${ }^{1}$ Associate Professor, Department of Radiology, Osmania Medical College/Modern Government Maternity Hospital, Hyderabad. \\ 2Professor, Department of Radiology, Osmania Medical College/Niloufer Hospital, Hyderabad. \\ 3Professor, Department of Radiology, Osmania Medical College/MNJ Cancer Hospital, Hyderabad.
}

\begin{abstract}
BACKGROUND

The key element, iodine set the stage for all future developments in contrast material for radiographic imaging. From this stage, extensive development of the quality of contrast medium has grown immensely to the present stage of non-ionic contrast media which are considered to be safe.

The aims of this study are-

1. To study the efficacy of static MR urography in comparison with conventional urography.

2. To study the levels and causes of obstruction of urinary tract by MR urography and compare them with other investigation procedures.
\end{abstract}

\section{MATERIALS AND METHODS}

120 patients who presented with clinical symptoms and signs pertaining to renal diseases and were positive on conventional MRI from Department of Radiodiagnosis, Osmania Medical College/ Hospital, Hyderabad, were selected for this study. This is an Institution-based cross-sectional study. MRU was performed on SIGNA HD 1.5 Tesla MRI, GE Healthcare (Milwaukee, USA). A body coil and commercially available software were used. SIGNA HD 1.5T is a diagnostic imaging device, which generates and displays transverse, sagittal, coronal and paraxial whole body images. Before performing MR urography, 3-plane localiser obtained in three planes was used to confirm optimum patient positioning; axial and coronal T2 weighted single shot FSE sequences of KUB region were taken, (TR $1615 \mathrm{msec}$, TE $88 \mathrm{msec}$, FOV 38 x 38, Matrix 256 x 256, NEX 0.56, TA 49 sec). The imaging method was based on the physical principle of magnetic resonance (MR). During the measurement, the patient was placed on the strong homogeneous magnetic field. The hydrogen nuclei (protons) distributed throughout the entire body tissue generate signals when stimulated by an RF field. These signals were processed into images by a computer.

\section{RESULTS}

In our study, 3D respiratory triggered sequences 3D FR FSE (Fast Recovery Fast Spin Echo) sequence was used. It provides high signal intensity of fluid even with short repetition time. After signal decay at end of echo train, a negative 90 degree pulse align spins with long T2 from transverse plane to longitudinal plane leading to a much faster recovery of tissues with long T2 time to equilibrium and thus better contrast between tissues with long and short T2. A frequency-selective fat-saturation pulse was used to reduce signal from retroperitoneal fat. During a period of 3 years, a total of 120 patients formed the study group. This included 52 females (43\%) and 68 males (57\%). Most patients in this study group belonged to the $3^{\text {rd }}$ decade. Commonest complaint in this study was pain followed by dysuria. MR Urography images were of relatively good quality. Distension of urinary bladder aided in better visualisation of upper urinary tract. We had considerable difficulty in removal of overlying bowel from images of few patients. Renal sizes could be easily measured, renal contours were normal, no masses and other parenchymal abnormalities were seen. Calyceal, forniceal and infundibular anatomy was not seen with the same detail as with an IVU. Anatomy of retroperitoneal and adjacent organs were reasonably seen.

\section{CONCLUSION}

In conclusion, MR urography provides high quality imaging of urinary tract and is an accurate and safe diagnostic alternative to other urological procedures. This when combined with excretory MRU, conventional MR images and MRA in a single session can yield a rapid and complete diagnostic evaluation of urinary tract and have the potential to provide the same information as can be obtained with multiple separate diagnostic studies.

\section{KEYWORDS}

MR Urography, 3D FRFSE Sequence, Caliceal, Forniceal Abnormalities, Hydronephrosis, Calculus, Intraluminal, Contrast Opacification, Functioning and Non-Functioning Kidneys, Polyuria, Dysuria.

HOW TO CITE THIS ARTICLE: Jyothi JSA, Kumari MV, Jayalatha N. Non-contrast magnetic resonance urography- a prospective study. J. Evolution Med. Dent. Sci. 2017;6(86):5975-5980, DOI: 10.14260/jemds/2017/1300

'Financial or Other Competing Interest': None.

Submission 04-06-2017, Peer Review 13-10-2017,

Acceptance 19-10-2017, Published 26-10-2017.

Corresponding Author:

Dr. J. S. Aswini Jyothi,

Flat: 204, Vasavi Bhuvana Apartments,

H. No: 8-3-981/1,3,4,6,8,10,11,

Srinagar Colony, Hyderabad-500037, Telangana.

E-mail: jyothijs@yahoo.com

DOI: $10.14260 /$ jemds $/ 2017 / 1300$

\section{BACKGROUND}

Conrad Roentgen discovered X-rays in 1895. Early attempts at opacification of urinary tract included retrograde placement of ureteral intraluminal wires and opaque catheters, air, colloidal silver and sodium iodide. Iodinated contrast agents that were excreted by kidney and could be administered intravenously were developed in 1920s.

The key element, iodine set the stage for all future developments in contrast material for radiographic imaging. From this stage, extensive development of the quality of 
contrast medium has grown immensely to the present stage of non-ionic contrast media, which are considered to be safe. New generation of contrast media for radiographic visualisation appears to be peaked, unlikely to be surpassed in the near future.

For a long time, IVU has been gold standard for the detection of dilatation of urinary tract. Over the years, the use of IVU has become somewhat less due to the advent of crosssectional imaging, but nevertheless remained in general use because of its low cost. IVU has the disadvantage of using ionising radiation and iodinated contrast media, both of which contribute to some extent to morbidity and mortality. ${ }^{1}$ The use of ionising radiation may also be an issue, particularly in children, young adults and obviously during pregnancy.

Ultrasonogram has significantly reduced the referrals for IVU as it is a safe, non-invasive method of assessment of urinary tract dilatation, but visualisation of non-dilated collecting system needs contrast opacification. ${ }^{2}$ However, above mentioned non-ionic contrast media cannot be administered in patients with poor renal function.

MR phenomenon was discovered by Bloch and Purcell for which they were awarded Nobel prize for Physics in 1952. Paul Christian Lauterbur along with Peter Mansfield pioneered use of MRI for medical imaging, for which they were awarded Nobel prize for Physiology/ Medicine in 2003.

MRU is becoming an integral part of recently emerging advances that involve clinical radio-urology. The advantages of MRU are that it is a non-invasive technique, allows multiplanar imaging, good soft tissue contrast and resolution and there is no need for contrast agents. So, it is useful for imaging of non-functioning kidneys. There is lack of ionising radiation with this procedure, so it is useful in children during pregnancy ${ }^{3}$ and in those who require repeated examination of urinary tract. Initially, Rapid Acquisition with Relaxation Enhancement technique (RARE) was introduced by Hennig ${ }^{4,5}$ et al in 1986 that facilitated the visualisation of collecting system without administration of intravenous contrast. Even faster technique for MR urography, which allowed ultrafast acquisition on T2 weighted high resolution called HASTE6,7 (Half-Fourier single-shot turbo spin-echo) (Siemens: Erlangen, Germany). This technique depends on intrinsically high SI of urine for contrast and therefore no IV contrast is administered. Renal parenchyma and urinary tract can be visualised in few seconds reducing motion artefacts. In the present study, 3-Dimensional FSE sequences are employed.

This study is an attempt to know and understand the diagnostic capability of urography and its efficacy in the visualisation of urinary collecting system.

\section{Aims}

1. To study the efficacy of static MR urography in comparison with conventional urography.

2. To study the levels and causes of obstruction of urinary tract by MR urography and compare them with other investigation procedures.

\section{Study Plan}

120 patients were selected for this study, who presented with clinical symptoms and signs pertaining to renal diseases and were positive on conventional MRI.

\section{Inclusion Criteria}

a) Any patients with clinical symptoms of urinary tract disease (obstructive and non-obstructive disease).

b) Previous history of contrast allergy.

c) Renal failure.

d) Poorly functioning kidneys where contrast is contraindicated.

\section{Exclusion Criteria}

a) Cardiac Pacemaker.

b) Metallic implants.

c) Claustrophobia.

d) Non-cooperative patient.

e) Excessive fluid in bowel loops.

\section{MATERIALS AND METHODS}

120 patients who presented with clinical symptoms and signs pertaining to renal diseases and were positive on conventional MRI from Department of Radiodiagnosis, Osmania Medical College/ OGH, Hyderabad, were selected for this study. This is an institution-based cross-sectional study.

MRU was performed on SIGNA HD 1.5 Tesla MRI, GE Healthcare (Milwaukee, USA).

A body coil and commercially available software were used. MRU was preferably performed on empty stomach to avoid fluid filled bowel loops. No other preparation was required.

SIGNA HD $1.5 \mathrm{~T}$ is a diagnostic imaging device which generates and displays transverse, sagittal, coronal and paraxial whole-body images. Before performing MR urography, 3-plane localiser obtained in three planes were used to confirm optimum patient positioning; axial and coronal T2 weighted single shot FSE sequences of KUB region were taken. (TR $1615 \mathrm{msec}$, TE $88 \mathrm{msec}$, FOV 38 x 38, Matrix 256 × 256, NEX 0.56, TA $49 \mathrm{sec}$ ).

The imaging method is based on the physical principle of magnetic resonance (MR).

During the measurement, the patient is placed on the strong homogeneous magnetic field. The hydrogen nuclei (protons) distributed throughout the entire body tissue, generate signals when stimulated by an RF field. These signals are processed into images by a computer.

\section{Patient Presentation}

Ureteric Colic, Polyuria, Dysuria, Haematuria, Fever.

\section{Technique of MRU}

Before performing MR urography, 3-plane localiser obtained in three planes was used to confirm optimum patient positioning; axial and coronal T2 weighted single shot FSE sequences of KUB region were taken. (TR $1615 \mathrm{msec}$, TE 88 msec, FOV 38 x 38, Matrix 256 x 256, NEX 0.56, TA 49 sec).

MR urography was done on 1.5 Tesla machine. Static fluid MR urography ${ }^{8}$ treats urinary tract as static column of fluid. MRU sequences are very heavily T2WI. The static fluid MRU technique closely resembles those used for T2W MRCP. 3Dimensional respiratory triggered sequences can be used to obtain thin section data sets that can be post processed to create VR/MIP images of entire urinary tract.9,10

FRFSE (Fast recovery fast spin echo) ${ }^{11}$ provides high signal intensity of fluid even with short repetition time and can be used for short breath hold techniques or respiratory 
gating. After signal decay at end echo train, a negative $90^{\circ}$ pulse align spins with $\mathrm{T} 2$ from transverse plane to longitudinal plane, leading to a much faster recovery of tissues with long T2 time to equilibrium and thus better contrast between tissues with long and short T2. It also has advantage for volumetric imaging. Fat saturation pulse was used to reduce high signal intensities from retroperitoneal fat.

Breath holding is major source of phase related artifact that degrades the quality of abdominal MR image. FR FSE T2 weighted images have an advantage over FSE T2WI, that they decrease phase related artifact. So it can be used in children who are unable to co-operate with breath holding instructions.

Respiratory triggering (RTr) can be used to decrease respiratory artifacts by synchronising image data collection with respiratory cycle.

Heavily T2W static fluid MR urograms resemble conventional excretory urograms and are useful for quickly identifying the level of UT obstruction. As the urographic effect is independent of renal excretory function it is useful for demonstrating the collecting system of an obstructed, poorly functioning kidney. This is the most important advantage of MRU over conventional IVU. MRU can be performed in children and useful for visualisation of urinary tract disorders in pregnant women.

Normal and abnormal fluid filled structures can interfere with static fluid MRU and the T2W sequences used to display urinary tract are not specific for urine.

IV hydration employed prior to technique in patient with non-distended collecting system. Alternatively, acquisition plane at post processing reconstruction volumes can be adjusted to exclude bowel or other fluid containing structures.

Sensitivity encoding parallel imaging techniques ASSET (array spatial sensitivity encoding technique) reduced imaging time and potential for respiratory motion artefacts.

\section{Technical Parameters}

M 3D/FRFSE- XL90 TR: 3750 msec.

TE: 620.9 msec.

FOV: 32 x 32 Matrix: 256 x 256 Thickness: 1/60-80 (mm) 1.6 THK Scan time - 2 mins 16 sec NEX: 0.75 .

If $3 \mathrm{D}$ acquisition is used, the acquisition time lengthens, but post processing function MIP can allow reconstructions from multiple slides.

\section{RESULTS}

This present study included 120 patients over a period of 3 years from Department of Radiodiagnosis, Osmania Medical College/ Hospital, Hyderabad. This is an institution-based cross-sectional study.

\begin{tabular}{|c|c|c|}
\hline No. of Patients & Male & Female \\
\hline 120 & 68 & 52 \\
\hline Percentage & $57 \%$ & $43 \%$ \\
\hline \multicolumn{2}{|c|}{ Table 1. Sex Distribution } \\
\hline
\end{tabular}

Out of 120 patients who underwent MR urography, 68 were males and 52 were females.

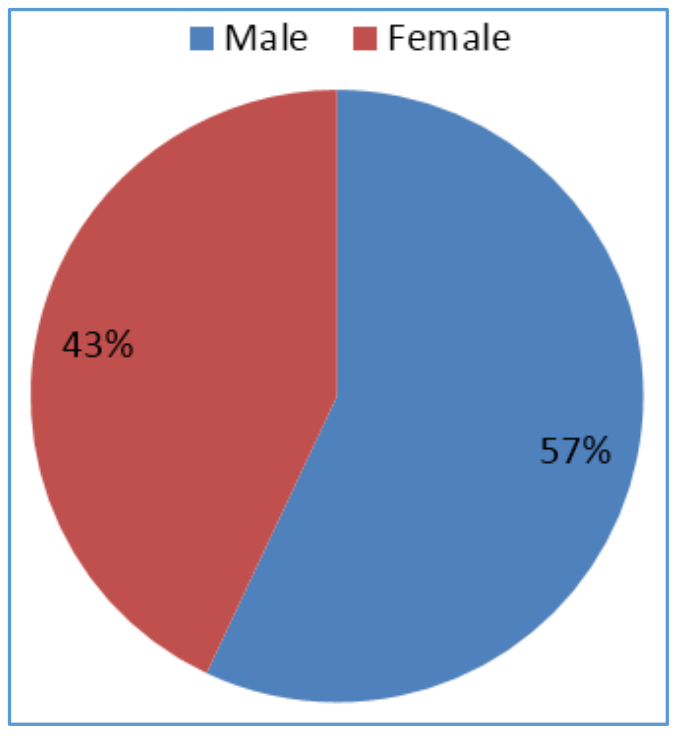

Figure 1

\begin{tabular}{|c|c|c|c|}
\hline Sl. No. & Age Group (Yrs.) & Incidence & Percentage \\
\hline 1. & $11-20$ & 16 & $13 \%$ \\
\hline 2. & $21-30$ & 28 & $23 \%$ \\
\hline 3. & $31-40$ & 48 & $40 \%$ \\
\hline 4. & $41-50$ & 12 & $10 \%$ \\
\hline 5. & $51-60$ & 8 & $7 \%$ \\
\hline 6. & $61-70$ & 8 & $7 \%$ \\
\hline \multicolumn{4}{|c|}{ Table 2. Age Incidence } \\
\hline
\end{tabular}

Most common age group was $3^{\text {rd }}$ decade followed by $2^{\text {nd }}$ decade.

\begin{tabular}{|c|c|c|}
\hline Sl. No. & Clinical Features & No. of Patients \\
\hline 1. & Pain & 40 \\
\hline 2. & Dysuria & 30 \\
\hline 3. & Haematuria & 26 \\
\hline 4. & Fever & 24 \\
\hline \multicolumn{3}{|c|}{ Table 3. Clinical Features } \\
\hline
\end{tabular}

Most common clinical presentation was pain followed by dysuria.

\begin{tabular}{|c|c|c|c|}
\hline Sl. No. & Site of Obstruction & No. of Cases & Percentage \\
\hline 1. & Renal pelvis & 20 & $17 \%$ \\
\hline 2. & Ureter & & \\
\hline & a) Proximal & 16 & $13 \%$ \\
\hline & b) Middle & 16 & $13 \%$ \\
\hline & c) Distal & 40 & $33 \%$ \\
\hline 3. & Bladder & 8 & $7 \%$ \\
\hline 4. & Normal & 20 & $17 \%$ \\
\hline
\end{tabular}

The commonest site of obstruction in this study was lower ureter followed by renal pelvis. 


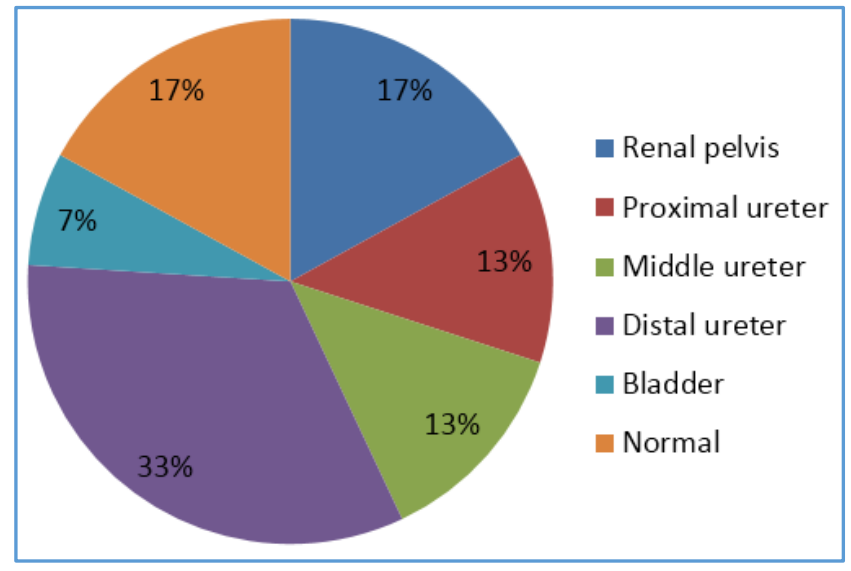

Figure 2

\begin{tabular}{|c|c|c|c|}
\hline Sl. No. & Findings & No. of Patients & Percentage \\
\hline 1. & Calculus & 72 & $65 \%$ \\
\hline 2. & Stricture & 8 & $7 \%$ \\
\hline 3. & Cystitis & 4 & $3 \%$ \\
\hline 4. & $\begin{array}{c}\text { Bladder } \\
\text { Diverticulum }\end{array}$ & 4 & $3 \%$ \\
\hline 5. & $\begin{array}{c}\text { Bladder Outlet } \\
\text { Obstruction }\end{array}$ & 8 & $7 \%$ \\
\hline 6. & Renal Agenesis & 4 & $3 \%$ \\
\hline 7. & Normal & 20 & $17 \%$ \\
\hline \multicolumn{3}{|c|}{ Table 5. Pathological Findings } \\
\hline
\end{tabular}

The most common pathological finding was calculus.

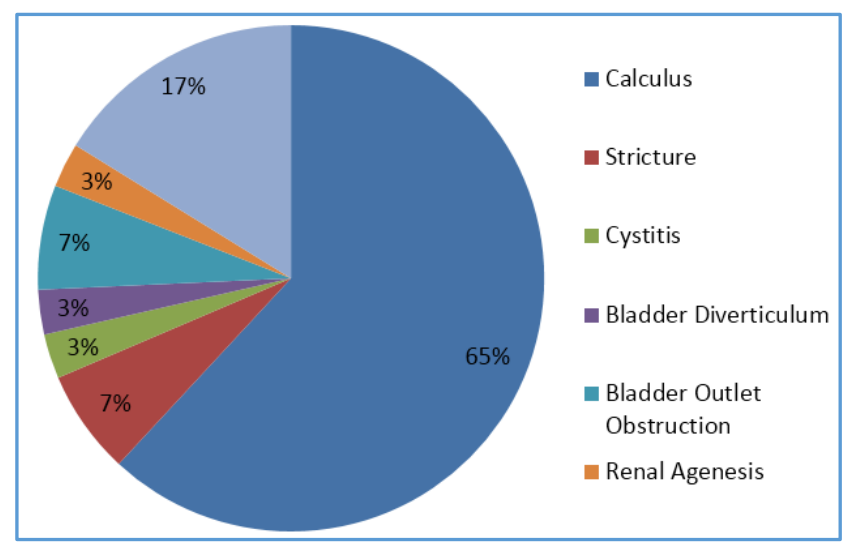

Figure 3

\begin{tabular}{|c|c|c|c|c|}
\hline Sl. No. & $\begin{array}{l}\text { Level of } \\
\text { Findings }\end{array}$ & $\begin{array}{c}\text { MR } \\
\text { Urography }\end{array}$ & IVU/USG & Accuracy \\
\hline 1. & Renal pelvis & 20 & 20 & $100 \%$ \\
\hline 2. & Ureter & & & \\
\hline & a) Proximal & 16 & 16 & $100 \%$ \\
\hline & b) Middle & 16 & 16 & $100 \%$ \\
\hline & c) Lower & 32 & 40 & $80 \%$ \\
\hline 3. & Bladder & 8 & 8 & $100 \%$ \\
\hline 4. & Normal & 24 & 20 & $83 \%$ \\
\hline
\end{tabular}

\begin{tabular}{|c|c|c|c|c|}
\hline Sl. No. & $\begin{array}{c}\text { Pathological } \\
\text { Findings }\end{array}$ & $\begin{array}{c}\text { MR } \\
\text { Urography }\end{array}$ & IVU/USG & Accuracy \\
\hline 1. & Calculus & 72 & 60 & $83 \%$ \\
\hline 2. & Stricture & 8 & 12 & $75 \%$ \\
\hline 3. & $\begin{array}{c}\text { Bladder } \\
\text { Diverticulum }\end{array}$ & 4 & 4 & $100 \%$ \\
\hline 4. & Cystitis & 4 & 4 & $100 \%$ \\
\hline 5. & $\begin{array}{c}\text { Bladder Outlet } \\
\text { Obstruction }\end{array}$ & 8 & 8 & $100 \%$ \\
\hline 6. & Renal Agenesis & 4 & 4 & $100 \%$ \\
\hline 7. & Normal & 24 & 20 & $83 \%$ \\
\hline \multicolumn{4}{|c|}{$\begin{array}{r}\text { Table 7. MR Urography Correlation } \\
\text { for Pathological Findings }\end{array}$} \\
\hline
\end{tabular}

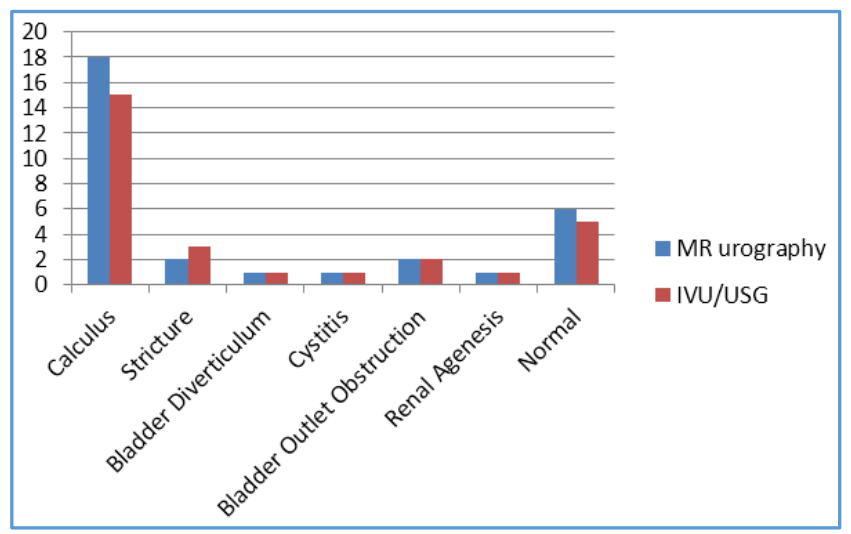

Figure 4

Figure 6: In graph, scale is $1=4$

\section{DISCUSSION}

Hydroureteronephrosis is a frequent urological problem with multiple aetiologies. In routine practice, USG and IVU are frequently used to diagnose hydronephrosis. For a long time IVU has been the only technique used in evaluating possible ureterohydronephrosis, but requires ionising radiation, iodinated contrast media and most important of all the functioning kidney.

Ultrasound was shown to be quite effective in diagnosis with a reported sensitivity of $98 \%^{1}$; however, the specificity is very low especially in depicting ureter. ${ }^{2}$ MRU can be used to evaluate urinary tract without ionising radiation and iodinated contrast material.,312 In the past, most authors used RARE (Rapid Acquisition Relaxation Enhancement), first described by Hennig et al in 1986.4,5 Heavily T2 weighted turbo spin echo pulse sequences were used to obtain water images of urinary tract. However, these techniques were time consuming and spatial resolution was poor. Moreover, only urinary tract was seen, but renal parenchyma was poorly visualised/ absent.

HASTE (Half-Fourier Acquisition Single-Shot Turbo Spin Echo) (Siemens; Erlangen, Germany) on SS FSE (Single-shot fast spin echo) (GE Health Systems) is a susceptibility insensitive single shot sequence, allowing ultrafast acquisition of T2 weighted high resolution images.7,13 Only half of the $\mathrm{K}$ space is measured, other half was constructed by half fourier. This sequence has the advantage of visualisation of urinary tract and renal parenchyma and can be obtained in a few seconds, thus reducing motion artifacts. 
In our study, 3D respiratory triggered sequences 3D FR FSE (Fast Recovery Fast Spin Echo) sequence was used. It provides high signal intensity of fluid even with short repetition time. After signal decay at end of echo train, a negative 90-degree pulse align spins with long T2 from transverse plane to longitudinal plane leading to a much faster recovery of tissues with a long $\mathrm{T} 2$ time to equilibrium and thus better contrast between tissues with long and short T2. Frequency selective, fat saturation pulses was used to reduce signal from retroperitoneal fat.

A total of 120 patients formed the study group. This included 52 females (43\%) and 68 males (57\%).

Most patients in this study group belonged to the $3^{\text {rd }}$ decade. Commonest complaint in this study was pain followed by dysuria.

MR Urography images were of relatively good quality. Distension of urinary bladder aided in better visualisation of upper urinary tract.14 We had considerable difficulty in removal of overlying bowel from images of few patients. Renal sizes could be easily measured, renal contours were normal, no masses or other parenchymal abnormalities were seen. Calyceal, forniceal and infundibular anatomy was not seen with the same detail as with an IVU. Anatomy of retroperitoneal and adjacent organs were reasonably seen.

\begin{tabular}{|c|c|c|c|}
\hline Sl. No. & Study & Sensitivity & Specificity \\
\hline 1. & ${\text { Catherine Roy et al }{ }^{12}}^{9}$ & $100 \%$ & $100 \%$ \\
\hline 2. & ${\text { Fintan Regan et }{ }^{9}}^{1}$ & $100 \%$ & $100 \%$ \\
\hline 3. & Martin O'Malley et al ${ }^{15}$ & $100 \%$ & $100 \%$ \\
\hline 4. & $\begin{array}{c}\text { Current Study MRU } \\
\text { with T1, T2 }\end{array}$ & $100 \%$ & $100 \%$ \\
\hline \multicolumn{3}{|c|}{$\begin{array}{c}\text { Table 8. Comparison with other Studies } \\
\text { Demonstrating Urinary Tract Dilatation }\end{array}$} \\
\hline
\end{tabular}

MR urography findings were correlated with findings of IVU/ USG and final diagnosis was obtained in 112 patients. Followup could not be obtained in 8 patients.

The accuracy of MRI was good in identifying level of obstruction. USG has high sensitivity of detecting obstruction. Its specificity is reported to be as low as $26 \%{ }^{16}$ due to obscuration of ureter by overlying bowel gas. Proximal ureteric obstruction identified by MR urography in 16 patients was confirmed in all patients on further followup (100\% accuracy).

Mid ureteric obstruction was diagnosed in all 16 patients by MR urography. Four patients had a $3 \mathrm{~mm}$ calculus in lower end of ureter as shown by USG was missed by MR urography. MR urography showed calculi in 72 patients. Correlation was obtained in 60 out of 72 patients with $83 \%$ accuracy on further followup. This correlates with study of Martin et al, 1997, who found 70\% - 83\% accuracy in diagnosing urolithiasis.

\begin{tabular}{|c|c|c|}
\hline Sl. No. & Study & Sensitivity \\
\hline 1. & ${\text { Catherine Roy et } \text { al }^{9}}_{10}$ & $100 \%$ \\
\hline 2. & Yi Tang et al & $100 \%$ \\
\hline 3. & Current study MRU with T1 and T2 & $93 \%$ \\
\hline \multicolumn{3}{|c|}{ Table 9. MRU showed Correlation to Level of } \\
Obstruction in 26 out of 28 Patients (93\% Accuracy) \\
\hline
\end{tabular}

MR urography accurately diagnosed strictures in 2 cases, $12,1783 \%$ to $89 \%$ accuracy.
MR urography diagnosed a case of left renal agenesis, which was associated with Mullerian duct anomaly-didelphic uterus.

MRU showed transplant kidney ${ }^{18}$ with clear demonstration of pelvicalyceal system and ureter where calculus obstruction was suspected. Incidental renal cortical cysts were detected in 8 patients.

MR urography demonstrates renal calculi as filling defects. It was difficult to differentiate them from blood clots and tumours, for which T1 weighted images and contrast were necessary. ${ }^{15}$

\begin{tabular}{|c|c|c|}
\hline Sl. No. & Study & Sensitivity \\
\hline 1. & ${\text { Martin O'Malley et al }{ }^{10}}^{\prime}$ & $90 \%$ \\
\hline 2. & Fintan Regan et al $^{6}$ & $80 \%$ \\
\hline 3. & $\begin{array}{c}\text { Current study MRU with } \\
\text { T1WI, T2WI }\end{array}$ & $83 \%$ \\
\hline \multicolumn{2}{|c|}{ Table 10 } \\
\hline
\end{tabular}

MRU performed in a pregnant woman ${ }^{12,19,20}$ in the second trimester showed compression of mid ureter with tapering at the pelvic brim. Ureter below this level of compression is relatively collapsed.

MRU clearly demonstrates congenital anomalies in a patient with renal duplication, ${ }^{21}$ where IVU could not be done. Hydronephrotic upper moiety and site of ectopic ureteral insertion were clearly demonstrated. ${ }^{15}$

\section{Summary}

A total of 120 patients were subjected to MR urography examination. This included 68 male (57\%) and 52 female (43\%).

Patients in $3^{\text {rd }}$ decades were mostly affected. Pain was the commonest symptom followed by dysuria. The lower part of ureter showed the maximum findings in this study. Calculus disease was the most common MRU finding. Regarding the presence of urinary tract dilatation and site of obstruction, MRU was accurate in $100 \%$ and $93 \%$ of cases respectively.

3D respiratory triggered sequence, 3D FRFSE along with fat saturation was the technique used. MRU was optimum for patients with renal failure and those allergic to iodinated contrast agents. It is safe as it involves non-ionising radiation, a feature especially useful in children, pregnant women and those requiring repeated imaging evaluation of the urinary tract. Usually, no patient preparation is required. It is operator independent unlike ultrasonography and independent of renal function. It is useful for assessment of congenital anomalies, renal donors and complication posttransplant. In acute urolithiasis, MR urography is not very relevant compared to other modalities like CT. But in chronic stone disease, it can provide valuable information about urinary tract anatomy.

In our study there were certain limitations, limited availability, high cost, relatively long examination time, low spatial resolution compared to IVU and CTU; sensitivity to motion (breathing and ureteral peristalsis), inherent contraindications like patients with pacemakers, claustrophobia, relative insensitivity for calcification and ureteric calculi. It relies on detecting secondary signs like ureteral dilatation and perinephric fluid. Superimposed abdominal fluid collections obscure segments of urinary tract. 
MR urography cannot differentiate dilatation between obstructive hydroureteronephrosis and dilatation due to vesicoureteric reflux. Static MRU provides no functional information. This should be complimented with contrast/ excretory MRU.

\section{CONCLUSION}

In conclusion, MR urography provides high quality imaging of urinary tract and is an accurate and safe diagnostic alternative to other urological procedures. This when combined with excretory MRU, conventional MR images and MRA in a single session can yield a rapid and complete diagnostic evaluation of urinary tract and have the potential to provide the same information as can be obtained with multiple separate diagnostic studies.

\section{REFERENCES}

[1] Cronan JJ. Contemporary concepts in imaging urinary tract obstruction. Radiol Clin North Am 1991;29(3):527-42.

[2] Webb JA. Ultrasonography in the diagnosis of renal obstruction. Br Med J 1990;301(6758):944-6.

[3] Nolte-Emsting CC, Staatz G, Tacke J, et al. MR urography today. Abdom Imaging 2003;28(2):191209.

[4] Hennig J, Friedburg H, Strobel B. Rapid nontomographic approach to MR myelography without contrast agents. J Comput Assist Tomogr 1986;10(3):375-8.

[5] Hennig J, Nauerth A, Friedburg H. RARE imaging: a fast imaging method for clinical MR. Magn Reson Med 1986;3(6):823-33.

[6] Regan F, Bohlman ME, Khazan R, et al. MR urography using HASTE imaging in the assessment of ureteric obstruction. AJR Am J Roentgenol 1996;167(5):111520.

[7] Tang Y, Yamashita Y, Namimoto T, et al. The value of MR urography that uses HASTE sequences to reveal urinary tract disorders. AJR Am J Roentgenol 1996;167(6):1497-502.

[8] Leyendecker JR, Barnes CE, Zagoria RJ. MR urography: techniques and clinical applications. Radio Graphics 2008;28(1):23-46.

[9] Roy C, Saussine C, Jahn C, et al. Evaluation of RARE-MR urography in the assessment of ureterohydronephrosis. J Comput Assist Tomogr 1994;18(4):601-8.
[10] O'Malley ME, Soto JA, Yucel EK, et al. MR urography: evaluation of a three-dimensional fast spin-echo technique in patients with hydronephrosis. AJR Am J Roentgenol 1997;168(2):387-92.

[11] Huang IH, Emery KH, Laor T, et al. Fast-recovery fast spin-echo T2-weighted MR imaging: a free-breathing alternative to fast spin-echo in the pediatric abdomen. Pediatr Radiol 2008;38(6):675-9.

[12] Leyendecker JR, Barnes CE, Zagoria RJ. MR urography: techniques and clinical applications. Radiographics 2008;28(1):23-46.

[13] Nolte-Ernsting CC, Adam GB, Gunther RW. MR urography: examination techniques and clinical applications. Eur Radiol 2001;11(3):355-72.

[14] Colville JA, Killeen RP, Buckley O, et al. Does a full bladder aid upper tract visualization in magnetic resonance urography. Australas Radiol 2007;51(4):362-4.

[15] Leyendecker JR, Gianini JW. Magnetic resonance urography. Abdom Imaging 2009;34(4):527-40.

[16] Kamholtz RG, Cronan JJ, Dorfman GS. Obstruction and the minimally dilated renal collecting system: US evaluation. Radiology 1989;170(1 Pt 1):51-3.

[17] Shokeir AA, El-Diasty T, Eassa W, et al. Diagnosis of noncalcareous hydronephrosis: role of magnetic resonance urography and noncontrast computed tomography. Urology 2004;63(2):225-9.

[18] Schubert RA, Göckeritz S, Mentzel HJ, et al. Imaging in ureteral complications of renal transplantation: value of static fluid MR urography. Eur Radiol 2000;10(7):1152-7.

[19] Spencer JA, Chahal R, Kelly A, et al. Evaluation of painful hydronephrosis in pregnancy: magnetic resonance urographic patterns in physiological dilatation versus calculous obstruction. J Urol 2004;171(1):256-60.

[20] Grenier N, Pariente JL, Trillaud H, et al. Dilatation of the collecting system during pregnancy: physiologic vs obstructive dilatation. Eur Radiol 2000;10(2):271-9.

[21] Joshi MP, Shah HS, Parelkar SV, et al. Role of magnetic resonance urography in diagnosis of duplex renal system: our initial experience at a tertiary care institute. Indian J Urol 2009;25(1):52-5. 